\title{
Aspiration-Point-Based Multi-Objective Path Planning Method for an Unmanned Aerial Vehicle*
}

\author{
By Hiroki OMAGARI and Shin-Ichiro HigASHINO \\ Department of Aeronautics and Astronautics, Kyushu University, Fukuoka, Japan
}

(Received January 30th, 2017)

\begin{abstract}
This research presents a new multi-objective path planning method for an unmanned aerial vehicle (UAV) using evolutionary computation. The proposed method searches for a desirable Pareto-optimal solution using an "aspiration point" and an "ideal point." The aspiration point refers to the preference information for a decision maker (DM), and the ideal point represents a virtual solution that optimizes all objective functions simultaneously. All of the solutions generated in using evolutionary computation evolve toward the aspiration region, which is determined by the aspiration point. If a solution that is closer to the ideal point than the aspiration point is generated in the search process, the aspiration point is moved to the position of the solution point. This process is repeated until specific termination conditions are satisfied. Some results of the benchmark test problems show that the proposed method can efficiently generate the Pareto-optimal solution for the DM and a high probability compared to the existing method called the "weighted-sum method." The usefulness of the proposed method is also shown by applying it to a multi-objective path planning problem that assumes an aerial photo-shoot mission using a UAV.
\end{abstract}

Key Words: Unmanned Aerial Vehicle, Evolutionary Computation, Multi-Objective Optimization Path Planning, Aspiration Point

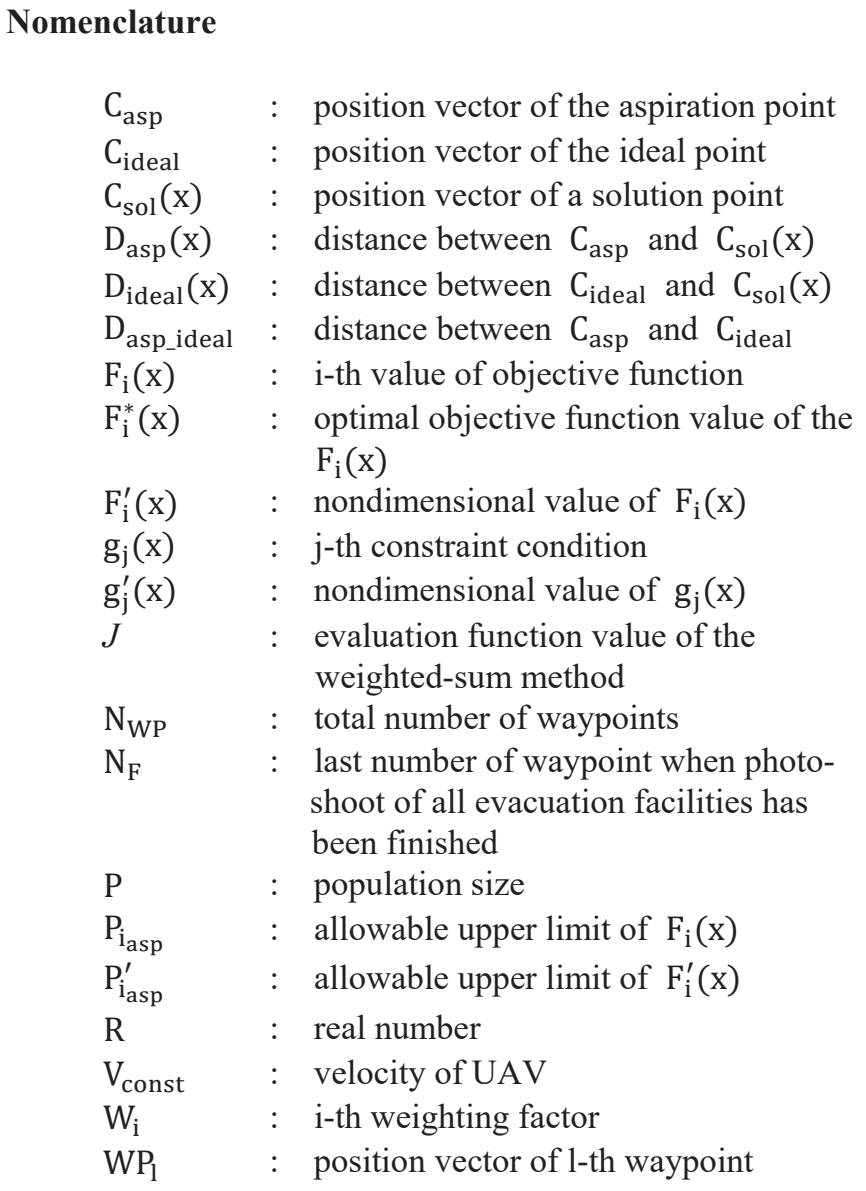

C) 2017 The Japan Society for Aeronautical and Space Sciences. *Presented at the 2016 Asia-Pacific International Symposium on Aerospace Technology (APISAT-2016), Oct. 25-27, 2016, Toyama, Japan

$\begin{array}{cl}\mathrm{x} & : \text { vector of design variables } \\ \mathrm{x}_{\mathrm{k}} & : \text { k-th component of design variable } \\ & : \text { vector } \\ X & : \text { feasible design space } \\ \mathrm{Z}_{\mathrm{l}} & \mathrm{z} \text {-component of } \mathrm{WP}_{\mathrm{l}} \\ \text { Subscripts } & : \text { aspiration point } \\ \text { asp } & : \text { constant value } \\ \text { const } & : \text { ideal point } \\ \text { ideal } & : \text { number of constraint conditions } \\ \mathrm{m} & : \text { number of objective functions } \\ \mathrm{M} & : \text { nolution point } \\ \mathrm{n} & : \text { waypoint } \\ \text { sol } & \end{array}$

\section{Introduction}

Recently, unmanned aerial vehicles (UAVs) have been increasingly deployed not only in military use, but also in civilian applications, such as for agricultural purposes, ${ }^{1)}$ infrastructure inspections, ${ }^{2)}$ life-saving missions, ${ }^{3,4)}$ delivery services, ${ }^{5,6)}$ and other purposes. ${ }^{7,8)}$ Many researchers are paying close attention to this field. Path planning is an indispensable technical element of UAV navigation. The $\mathrm{A}^{*}$ algorithm $^{9)}$ is known as the method that is guaranteed to provide an optimal solution that minimizes the path length using heuristics. Other conventional methods are based on the methods such as the potential field theory ${ }^{10)}$ and the Voronoi diagram. ${ }^{11)}$ These methods mainly focus on the generation of the shortest distance while considering obstacle avoidance. In contrast, the methods for solving multi-objective path planning problems, where two or more objective 
functions must be simultaneously optimized, have been less researched so far. For these reasons, the development of a multi-objective path planning method can be expected to enable an expanded range of UAV applications in the future. This study proposes a multi-objective path planning method that can be used for practical applications, such as an aerial photo-shoot mission.

The outline of this research is as follows. In Section 2, we introduce some studies related to evolutionary multi-objective optimization methods and briefly explain some drawbacks of these methods. In Section 3, we comprehensively describe the proposed method and show how to deal with the drawbacks of existing methods. In Section 4, we compare the proposed method with the "weighted-sum method" using benchmark test problems called "DTLZ" in order to evaluate its search performance. In Section 5, we formulate an aerial photo-shoot mission as a multi-objective path planning problem and generate the desirable flight path using the proposed method. The performance of the proposed method is compared with that of the weighted-sum method again. Finally, we conclude the research in Section 6.

\section{Background}

\subsection{Multi-objective optimization problem concept}

Typically, a multi-objective optimization problem can be formulated as follows: ${ }^{13}$ )

$$
\left\{\begin{array}{l}
\min _{x} F_{i}(x), \quad i=1,2, \ldots, M \\
\text { subject to } g_{j}(x) \leq 0, j=1,2, \ldots, m \\
x=\left[x_{k}, x_{k}, \ldots, x_{k}, \ldots, x_{k}\right]^{T}, \quad X=\left\{x \mid x \in R^{n}\right\}
\end{array}\right.
$$

Generally, no fully optimal solution exists that can simultaneously optimize all of the objective functions. The conceptual diagram of this idea is shown in Fig. 1. For this figure, it is assumed that two objective functions $\left(\mathrm{F}_{1}(\mathbf{x})\right.$ and $\left.\mathrm{F}_{2}(\mathbf{x})\right)$ must be optimized simultaneously. The region surrounded by the broken orange line indicates $X$. For example, when the two solutions $A$ and $B$ are compared, $\mathrm{F}_{1}(\mathbf{A})<\mathrm{F}_{1}(\mathbf{B})$ and $F_{2}(A)<F_{2}(B)$, so that $\mathbf{A}$ is superior to $\mathbf{B}$. This is referred to as "A dominates B." All dominated solutions are shown as yellow circles in Fig. 1. Meanwhile, when the two solutions, $\mathbf{A}$ and $\mathbf{C}$, are compared, $\mathrm{F}_{1}(\mathbf{A})<\mathrm{F}_{1}(\mathbf{C})$ and $\mathrm{F}_{2}(\mathbf{A})>\mathrm{F}_{2}(\mathbf{C})$, so that it is not possible to determine which of the solutions is superior. The relationship between these two solutions is called a "trade-off." The solutions that are not dominated by any other solutions such as this are called the "Pareto-optimal solutions." Usually, a large number of Pareto-optimal solutions exist for a multi-objective problem, as shown by the green circles drawn in Fig. 1. The mapping of all Pareto-optimal solutions forms a curve as shown by the green lines. The curve is commonly referred to as the Pareto-frontier ${ }^{14)}$. A decision maker (DM) must choose "a preferred solution" from these Pareto-optimal solutions. One example of a preferred solution is shown by the magenta circle.

\subsection{Related work}

We can obtain many solutions simultaneously using the evolutionary computation (EC) method that has been

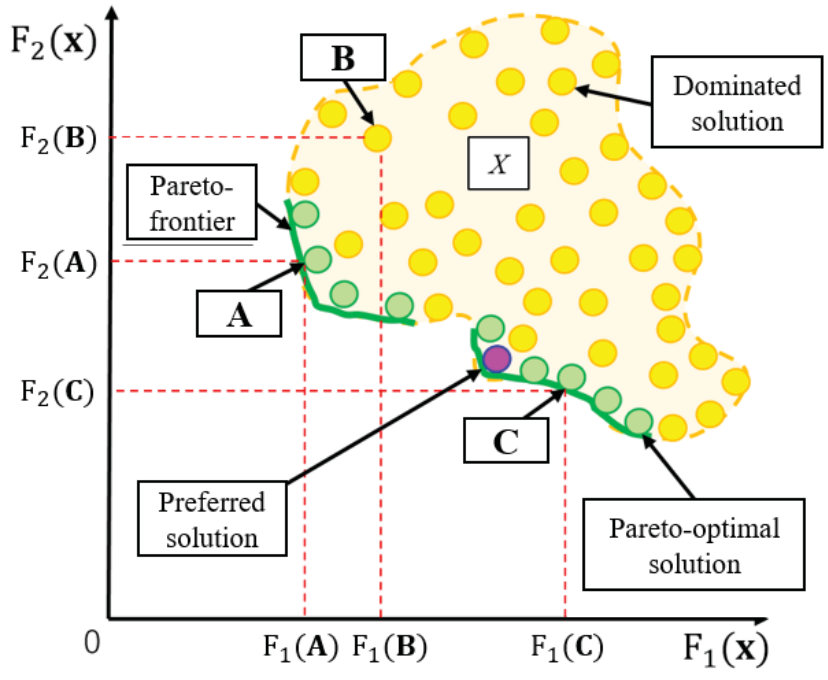

Fig. 1. Schematic of a multi-objective optimization problem and the Pareto-frontier.

frequently used to solve multi-objective optimization problems. This algorithm is based on Darwin's theory of evolution and uses operations known as natural selection, crossover, and mutation. This method searches for a sub-optimal solution that may be a satisfactory solution for the DM within a reasonable time rather than trying to find the exact optimal solution that would require a long time.

During the past few decades, various evolutionary multiobjective optimization methods has been proposed to find the preferred solution for the DM. For example, the Nondominated Sorting Genetic Algorithm II (NSGAII), ${ }^{15,16)}$ Strength Pareto Evolutionary Algorithm 2 (SPEA2) ${ }^{17)}$ and Multiple-Objective Genetic Algorithm (MOGA) ${ }^{18)}$ methods are widely known. These methods have demonstrated their usefulness in finding a well-converged and well-distributed set of the Pareto-optimal solutions. In fact, these evolutionary multi-objective optimization methods have been used in many practical applications such as manufacturing ${ }^{12)}$ engineering, ${ }^{13,20)}$ finance, ${ }^{19)}$ chemistry, ${ }^{20)}$ and so on. ${ }^{21)}$

Nevertheless, these evolutionary multi-objective optimization methods also have some drawbacks. For instance, a visualization method for four or more objective functions has not been established yet. This may limit the ability to find the entire non-dominated set of solutions. In addition, the selection pressure, which propels the solutions toward the Pareto-frontier, becomes weak because the proportion of Pareto-optimal solution in the solution space increases explosively when the number of objective functions increases. For this reason, the number of solutions that are not dominated by other solutions increases and it becomes difficult to select some individuals that should be taken over to the next generation. This becomes some factors that stagnate or worsen the evolution of the solutions.

Therefore, it is rational to focus the limited computational resources only on searching for the preferred region for the DM. An alternative approach is to treat the problem by converting it into a single objective function problem. The weighted-sum method is a typical method that follows this approach. It allows effective usage of the computational 
budget by converting the multi-objective optimization problem into a single-objective optimization problem, and can alleviate the selection pressure problem imposed on the dominance-based evolutionary algorithms. However, this method also suffers from some drawbacks. If some solutions exist alone in the non-convex regions of the Pareto-frontier, adjusting the weighting factors does not work well. In the worst case, this may result in the selection of inferior solutions instead of superior solutions in the non-convex regions. $^{22-24)}$

The goal programming method of Deb is another wellknown approach for solving this problem. ${ }^{25}$ This method requires the DM to specify a goal for each of the objective function values in advance. Deb modified the NSGA method to find a set of solutions that are the closest to the supplied level of the solutions. Unfortunately, this method did not focus on finding a Pareto-optimal solution corresponding to the multi-objective optimization problem, but rather attempted only to find a solution that satisfies the supplied goal given by the DM. Reference-point-based methods ${ }^{26,27)}$ exhibit these problems as well.

\section{Moving Aspiration-Point-Based Method}

To improve these weak points of the existing methods described above, we propose a new evolutionary multi-objective optimization algorithm named the "aspiration-pointbased method" for obtaining the preferred solution for the DM efficiently. The "aspiration point" means the preference information for the DM. The proposed method incorporates the advantages of the conventional methods and eliminates their drawbacks.

\subsection{Proposed method}

The proposed method consists of six steps. The conceptual diagrams are presented in Fig. 2. The diagram describes how to generate the preferred solution for the DM.

Step 1: Calculate the optimal value of objective functions.

The DM needs to calculate $\mathrm{F}_{\mathrm{i}}^{*}(\mathbf{x})$ respectively by optimizing the multi-objective optimization problem as the single-objective one of $\mathrm{F}_{\mathrm{i}}(\mathbf{x})$.

\section{Step 2: Normalize objective functions and define solution points.}

After generating a certain number of initial individuals, the DM normalizes $\mathrm{F}_{\mathrm{i}}(\mathbf{x})$ by corresponding $\mathrm{F}_{\mathrm{i}}^{*}(\mathbf{x})$ as follows:

$$
\mathrm{F}_{\mathrm{i}}^{\prime}(\mathbf{x})=\frac{\mathrm{F}_{\mathrm{i}}(\mathbf{x})}{\mathrm{F}_{\mathrm{i}}^{*}(\mathbf{x})}
$$

In the case where $\mathrm{F}_{\mathrm{i}}^{*}(\mathbf{x})$ becomes 0 , the DM needs to determine the appropriate representative value instead of $\mathrm{F}_{\mathrm{i}}^{*}(\mathbf{x})$ based on his preferred information in order to scale $F_{i}(\mathbf{x})$. This approach can be expected to reduce the computational load compared with handling the quantities in different units simultaneously, such as in the NSGAII or SPEA2 methods. Then, we define "solution points" according to Eq. (3) in order to locate the individuals on a dimensionless coordinate system.

$$
\mathrm{C}_{\mathrm{sol}}(\mathbf{x})=\left[\mathrm{F}_{1}^{\prime}(\mathbf{x}), \mathrm{F}_{2}^{\prime}(\mathbf{x}), \ldots, \mathrm{F}_{\mathrm{i}}^{\prime}(\mathbf{x}), \ldots, \mathrm{F}_{\mathrm{M}}^{\prime}(\mathbf{x})\right]^{\mathrm{T}}
$$

In Fig. 2 (a), yellow circles represent these solution points.

\section{Step 3: Define the ideal point and the aspiration point.}

We define two kinds of reference points named "ideal point" and "aspiration point", respectively. The ideal point is defined as

$$
\mathrm{C}_{\text {ideal }}=[1,1, \ldots, 1]^{\mathrm{T}}
$$

Where the ideal point means a virtual solution that optimizes all of the objective functions at the same time. In the case where all $\mathrm{F}_{\mathrm{i}}^{*}(\mathbf{x})$ are not 0 , each component of the ideal point is derived by substituting $\mathrm{F}_{\mathrm{i}}^{*}(\mathbf{x})$ into the numerator of Eq. (2). In the case where $F_{i}^{*}(\mathbf{x})$ becomes 0 , the DM sets the component of the ideal point that corresponds to the objective function to 0 instead of 1 . The ideal point is shown as a red circle in Fig. 2 (a).

Next, we assume that some constraint conditions are given by

$$
\begin{gathered}
g_{j}(x)=F_{i}(x)-P_{i_{a s p}} \leq 0, \\
i=1,2, \ldots, M, \quad j=1,2, \ldots, m
\end{gathered}
$$

Where $\mathrm{P}_{\mathrm{asp}}$ means the upper limit of the allowable value as a preferred solution for the DM. The constraint conditions can be rewritten by normalizing both sides using Eq. (2).

$$
\begin{gathered}
g_{j}^{\prime}(x)=F_{i}^{\prime}(\mathbf{x})-P_{i_{a s p}}^{\prime} \leq 0, \\
i=1,2, \ldots, M, \quad j=1,2, \ldots, m
\end{gathered}
$$

Here, we also define the aspiration point using $\mathrm{P}_{\mathrm{i}_{\text {asp }}}^{\prime}$ as shown in Eq. (7).

$$
\mathrm{C}_{\mathrm{asp}}=\left[\mathrm{P}_{1_{\mathrm{asp}}}^{\prime}, \mathrm{P}_{\mathrm{assp}^{\prime}}^{\prime}, \ldots, \mathrm{P}_{\mathrm{asp}_{\mathrm{asp}}^{\prime}}^{\prime}, \ldots, \mathrm{P}_{\mathrm{M}_{\mathrm{asp}}}^{\prime}\right]^{\mathrm{T}}
$$

If those constraint conditions are not given in advance, the DM needs to determine the coordinates of the aspiration point using the preferred information. The priority of the objective function can be changed by adjusting the position of the aspiration point. For example, the DM can increase the priority of the objective functions by making the corresponding coordinates of the aspiration point close to the value of 1 .

As shown in Fig. 2 (a), blue dashed lines indicate the upper limits of the constraint conditions and the blue circle represents the aspiration point. The translucent purple surface represents the region surrounded by all coordinate axes and all perpendicular lines that are drawn from the aspiration point to each coordinate axis. We call this an "aspiration region." In Fig. 2 (a), it assumes that the aspiration point is located at $[3,1.7]^{\mathrm{T}}$. This means that the DM tolerates the deterioration of $\mathrm{F}_{1}(\mathbf{x})$ until $200 \%$ from $\mathrm{F}_{1}^{*}(\mathbf{x})$, and that of $\mathrm{F}_{2}(\mathbf{x})$ until $70 \%$ from $\mathrm{F}_{2}^{*}(\mathbf{x})$. In this case, the most suitable solution for the DM is the position indicated by the blue diamond-shaped marker that is the closest to the ideal point in the aspiration region. 


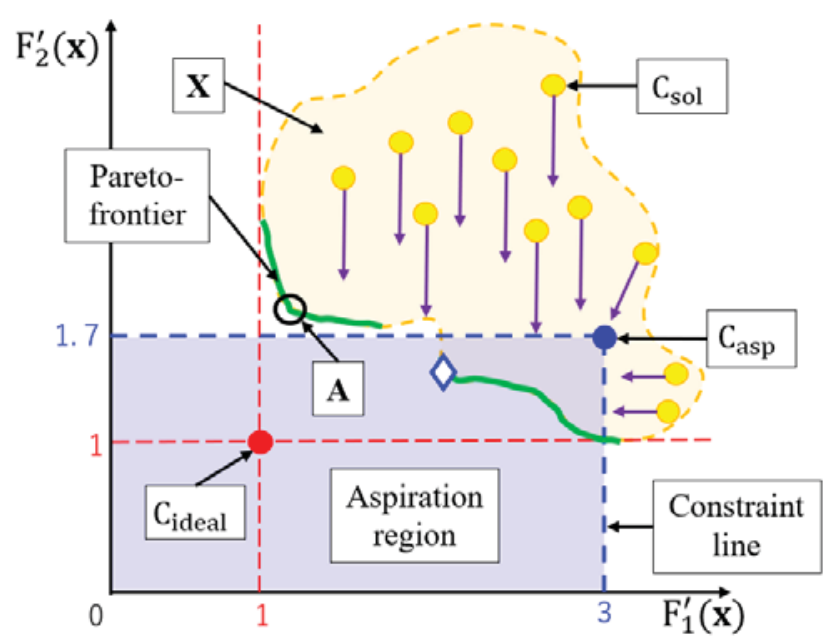

(a) Search process from Step 1 to Step 4 .

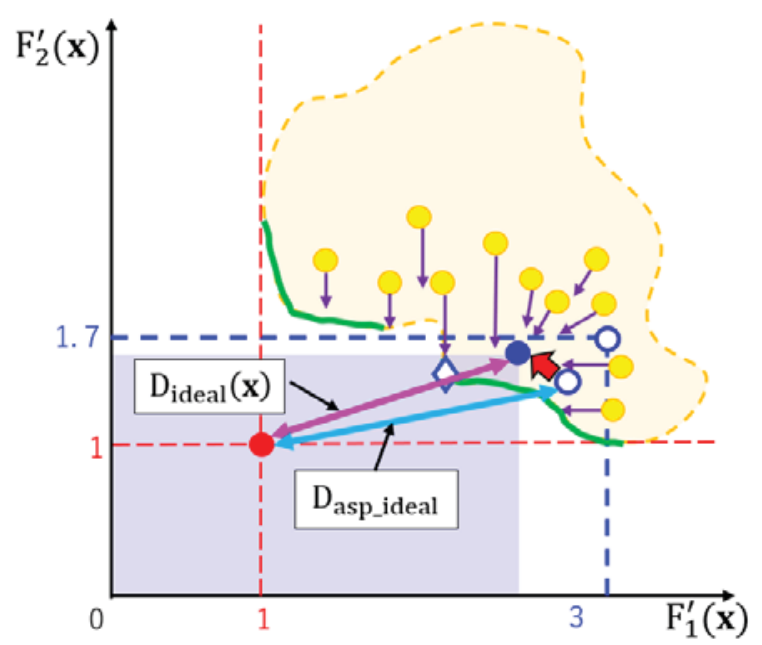

(c) Iteration of Step 2-Step 5 .

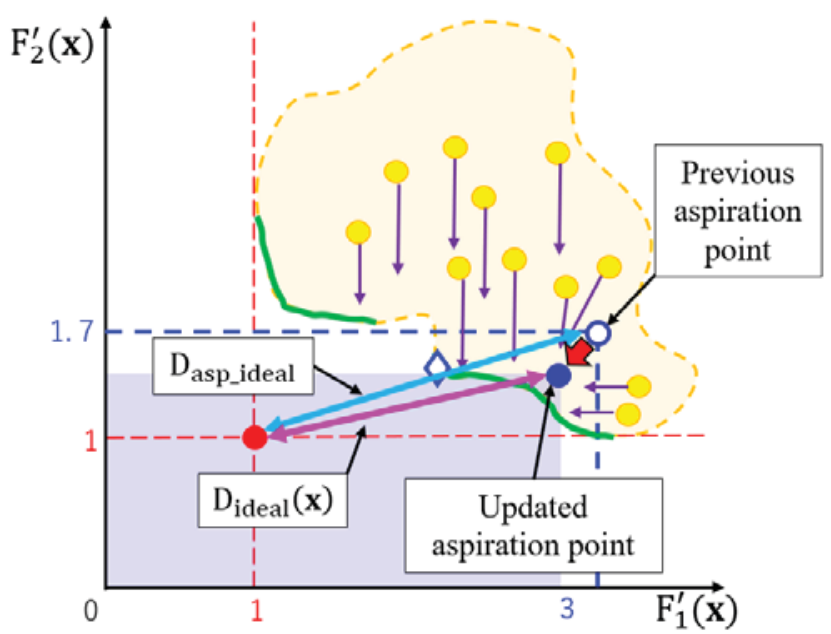

(b) Step 5 .

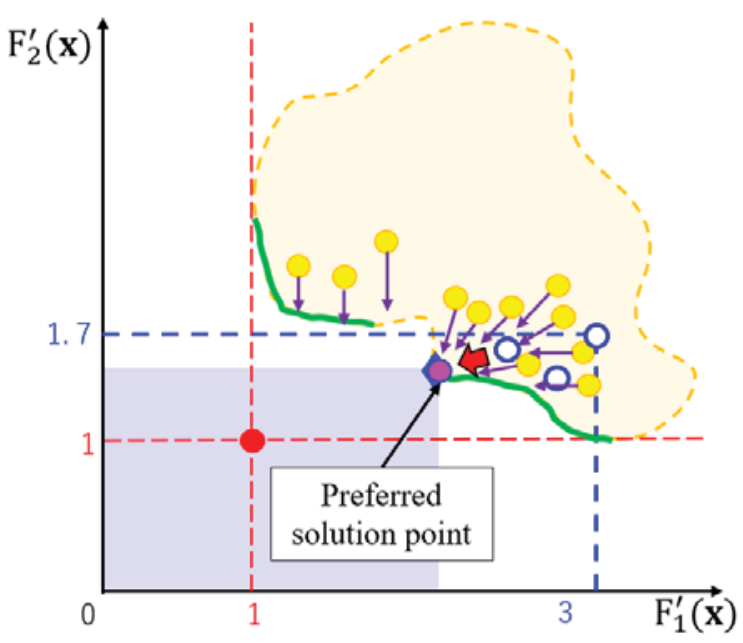

(d) Step 6 .

Fig. 2. Aspiration-point-based search process.

Step 4: Evolve all solutions towards the aspiration region.

In the proposed method, the solution points are evolved toward the aspiration region instead of the ideal point. The reason is that if the DM searches for a solution point which is closer to the ideal point, he obtains a solution point at the position indicated by A, as shown in Fig. 2 (a). It doesn't meet the constraint conditions. On the other hand, if the DM searches for a solution point that is the closer to the aspiration region, he can obtain a solution point in the aspiration region. From the above, we calculate Euclid distance between one of the solution points and the aspiration point, as shown in Eq. (8), and search for solution points that minimize this value in order to evolve all generated solution points toward the aspiration region.

$$
\mathrm{D}_{\mathrm{asp}}(\mathbf{x})=\left(\sum_{\mathrm{i}=1}^{\mathrm{M}}\left(\mathrm{F}_{\mathrm{i}}^{\prime}(\mathbf{x})-\mathrm{P}_{\mathrm{i}_{\text {asp }}^{\prime}}\right)^{2}\right)^{1 / 2}
$$

Where, if one of the $\mathrm{F}_{\mathrm{i}}^{\prime}(\mathbf{x})$ is smaller than $\mathrm{P}_{\mathrm{i} \text { asp }}^{\prime}$, it needs to be set $\mathrm{F}_{\mathrm{i}}^{\prime}(\mathbf{x})-\mathrm{P}_{\mathrm{i}_{\text {asp }}}^{\prime}$ to 0 in order to efficiently search for a solution point in the aspiration region.

Step 5: Move the aspiration point towards the ideal point.
We also calculate two other Euclid distances as follows:

$$
\begin{aligned}
& \mathrm{D}_{\text {asp_ideal }}=\left(\sum_{\mathrm{i}=1}^{\mathrm{M}}\left(1-\mathrm{P}_{\mathrm{i}_{\text {asp }}^{\prime}}^{\prime}\right)^{2}\right)^{1 / 2} \\
& \mathrm{D}_{\text {ideal }}(\mathbf{x})=\left(\sum_{\mathrm{i}=1}^{\mathrm{M}}\left(1-\mathrm{F}_{\mathrm{i}}^{\prime}(\mathbf{x})\right)^{2}\right)^{1 / 2}
\end{aligned}
$$

Where $\mathrm{D}_{\text {asp_ideal }}$ is the distance between the ideal point and the aspiration point, and $\mathrm{D}_{\text {ideal }}(\mathbf{x})$ is the distance between one of the solution points and the ideal point. The blue arrow represents $\mathrm{D}_{\text {asp_ideal }}$ and the magenta arrow represents $\mathrm{D}_{\text {ideal }}(\mathbf{x})$ in Figs. 2 (b) and 2 (c). If a solution point whose $\mathrm{D}_{\text {ideal }}(\mathbf{x})$ is less than $\mathrm{D}_{\text {asp_ideal }}$ is generated, we move the aspiration point to the solution point and substitute the values of $\mathrm{F}_{\mathrm{i}}^{\prime}(\mathbf{x})$ into $\mathrm{P}_{\mathrm{i}}^{\prime}$ asp in order to update the position of the aspiration point and $\mathrm{D}_{\text {asp_ideal. In this way, the new aspira- }}$ tion point is made closer to the blue diamond shaped marker than the previous aspiration point. Thus, it is expected that the next generation will be generated in the vicinity of the updated aspiration region.

Step 6: Repeat Step2-Step5.

This process is repeated until the termination condition is 
satisfied as shown in Fig. 2 (c). Finally, we can find the preferred solution on the Pareto-frontier that is closest to the ideal point in the aspiration region. Figure 2 (d) shows that the preferred solution drawn by the magenta circle is found at the same position as the blue diamond-shaped marker that is the most preferred solution for the DM.

\section{Evaluation using Benchmark Test Problems}

\subsection{Benchmark problem}

We use some scalable benchmark test problems known as "DTLZ"28) in order to evaluate the usefulness of the proposed method. The test problems can freely change $M$ and n. Additionally, the shape of the Pareto-frontier is known in advance, so that these test problems have been used frequently to evaluate the performance of multi-objective optimization methods. ${ }^{29)}$ In this section, we use the three benchmark test problems known as "DTLZ1", "DTLZ3", and "DTLZ7." Figure 3 shows the Pareto-frontier shape of the test problems in the case of $M=2$. As shown in this figure, the Pareto-frontiers have a linear shape in DTLZ1, a semicircle centered at the origin with a radius of 1 in DTLZ3, and are discontinuous in DTLZ7.

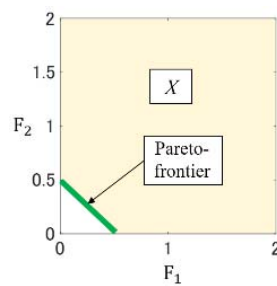

(a) DTLZ1.

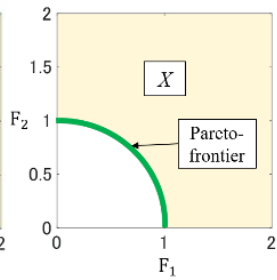

(b) DTLZ3.

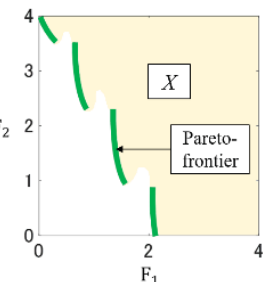

(c) DTLZ7.
Fig. 3. Pareto-frontier shapes of DTLZ test problems.

\subsection{Performance evaluation}

We applied the "elite preservation strategy"30) as a selection operation to take over the solution points, which minimize $\mathrm{D}_{\text {asp }}(\mathbf{x})$, for the next generation. In this way, the solutions that satisfy all of the constraint conditions and are closer to the ideal point can be obtained efficiently and have a high probability. We combined two methods for the crossover operation: we combined two methods, the "uniform crossover method"31) and the "one-point crossover"32) method. We also used a standard mutation operation in binary form. The search process was terminated when the maximum number of generations was reached. This simulation was calculated on a computer with a Core i7-4790 3.60GHz CPU having 8GB of RAM. MATLAB was used as the programming language. The other calculation conditions are shown in Table 1.

\begin{tabular}{|c|c|}
\hline Items & Values \\
\hline M & 2 \\
\hline $\mathrm{n}$ & 10 \\
\hline Number of individuals & 200 \\
\hline Mutation probability & 0.01 \\
\hline Crossover probability & 1 \\
\hline Max generation & 1000 \\
\hline
\end{tabular}

It is unnecessary to use Eq. (2) for the DTLZ problem because the objective functions are originally nondimensional values. Along with this, we can directly set arbitrary real numbers as the coordinates of the aspiration point. On the other hand, it is necessary to move the ideal point to the origin coordinates because the optimal values of all objective functions are 0 . Based on the above, we set the aspiration point to $[0.3,1.5]^{\mathrm{T}}$ for DTLZ1 and DTLZ3, and to $[1,3]^{\mathrm{T}}$ for DTLZ7. The ideal point was set to $[0,0]^{\mathrm{T}}$ for all of the test problems. Figures 4 (a), 4 (c) and 4 (e) show the state around the Pareto-frontier before the search, and Figures 4 (b), 4 (d) and 4 (f) show the results. Examination of the figures on the right-hand side shows that the aspiration point indicated by the blue circle is gradually approaching the Pareto-frontier along the red arrow and finally reaches the surface. Therefore, it was found that the proposed method can generate the preferred solution for the DM.

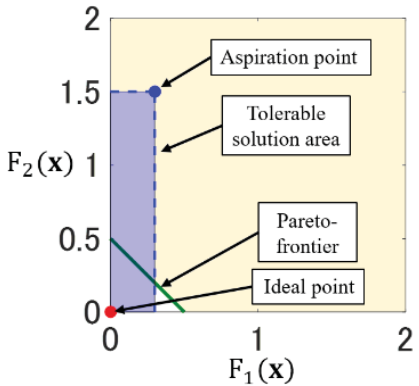

(a) Before search in DTLZ1.

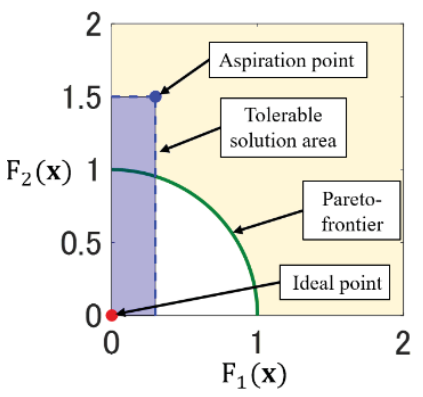

(c) Before search in DTLZ3.

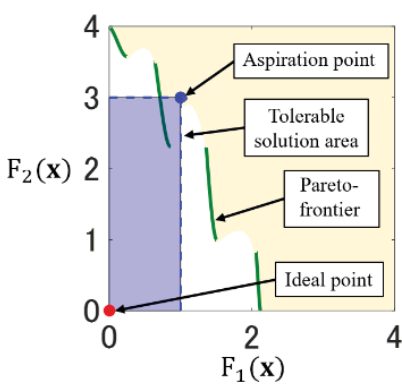

(e) Before search in DTLZ7

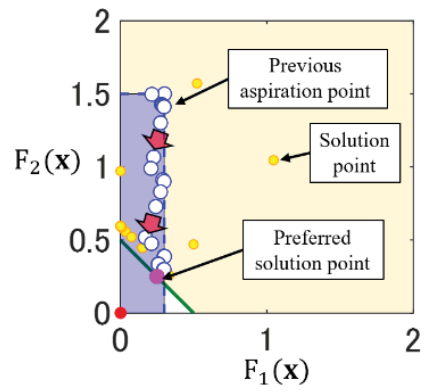

(b) After search in DTLZ1.

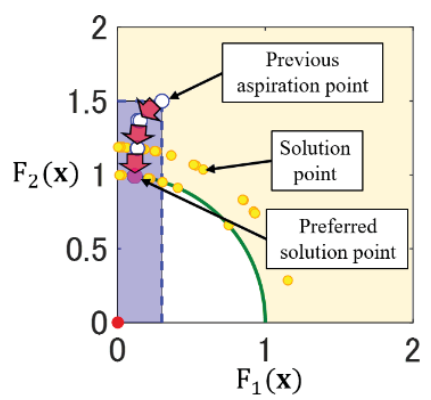

(d) After search in DTLZ3.

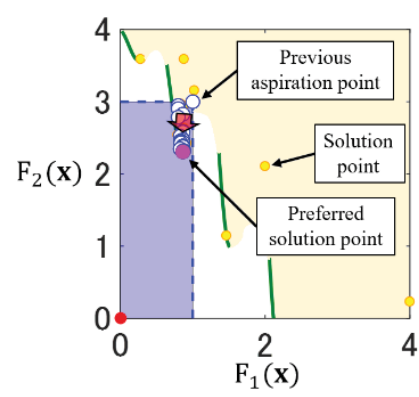

(f) After search in DTLZ7.
Fig. 4. Search results of the proposed method.

Next, we compared the search performance of the proposed method with that of the weighted-sum method because the weighted-sum method is often used for solving multi-objective optimization problems. The weighted-sum method can be formulated as follows:

$$
\left\{\begin{array}{c}
J=\sum_{i=1}^{\mathrm{M}} \mathrm{W}_{\mathrm{i}} \mathrm{F}_{\mathrm{i}}^{\prime}(\mathbf{x}) \\
\mathrm{W}_{\mathrm{i}}=\frac{1}{\mathrm{P}_{\mathrm{i}_{\mathrm{asp}}^{\prime}}}\left(1 / \sum_{\mathrm{i}=1}^{\mathrm{M}} \frac{1}{\mathrm{P}_{\mathrm{i} \text { sp }}^{\prime}}\right), \quad \sum_{\mathrm{i}=1}^{\mathrm{M}} \mathrm{W}_{\mathrm{i}}=1
\end{array}\right.
$$


where the multi-objective optimization is realized by minimizing $J$. The reason for deciding the weighting factors is that the value of $\mathrm{P}_{\mathrm{i}_{\text {asp }}}^{\prime}$ becomes smaller as the importance of $\mathrm{F}_{\mathrm{i}}^{\prime}(\mathbf{x})$ increases in the proposed method.

The performance tests were carried out by increasing the value of $\mathrm{M}$ from 2 to 5 in order to show the proposed method is effective even when the number of objective functions increases. To locate the aspiration point in the vicinity of the Pareto-frontier, the position of the aspiration point was determined from the range, as shown in Table 2. Here, it should be noted that if the aspiration point is set outside of $X$, it is impossible to conduct the performance tests because there is no solution in the aspiration region. Therefore, we assumed that the position of the aspiration point was limited within $X$. The other calculation conditions were the same as in the previous tests. The tests were repeated 100 times. The calculation results were as follows:

\begin{tabular}{cc} 
Table 2. Aspiration point range. \\
\hline DTLZ1 & $0.1 \sim 0.5$ \\
DTLZ3 & $0.1 \sim 1.0$ \\
DTLZ7 & $1.0 \sim 3.0$ \\
\hline
\end{tabular}

Figures 5 (a), 5 (c) and 5 (e) show the success ratio of generating a solution that meets the constraint conditions and that of generating a Pareto-optimal solution that meets the constraint conditions. The magenta bars represent the former, and the green bars represent the latter. As shown these figures, the success ratios of the proposed method were almost $100 \%$ in all cases, but that of the weighted-sum method were extremely low. Figures 5 (b), 5 (d) and 5 (f) show the average number of generations when a solution that meets the constraint conditions was generated and when a Pareto-optimal solution that meets the constraint conditions was generated. The black vertical lines represent the maximum and minimum number of generations. Examination of these figures shows that the proposed method is superior to the weighted-sum method in terms of generating the desired solution in all cases. The possible reasons why the average number of generations for the solution search in DTLZ7 was relatively small is that the region of $X$ is extremely small compared to the other DTLZ problems.
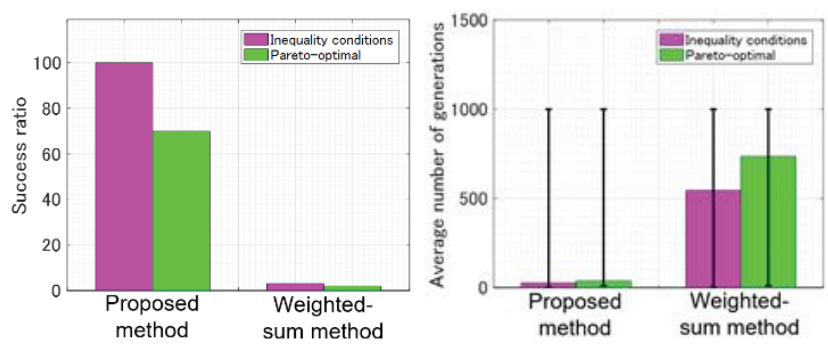

(a) Success ratio in DTLZ1

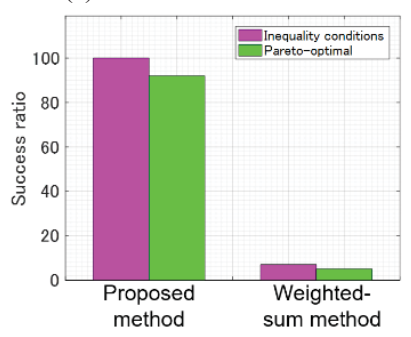

(c) Success ratio in DTLZ3.
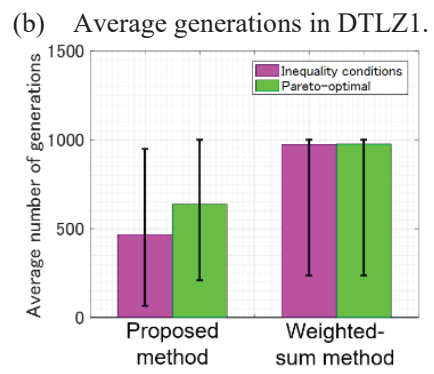

(d) Average generations in DTLZ3.
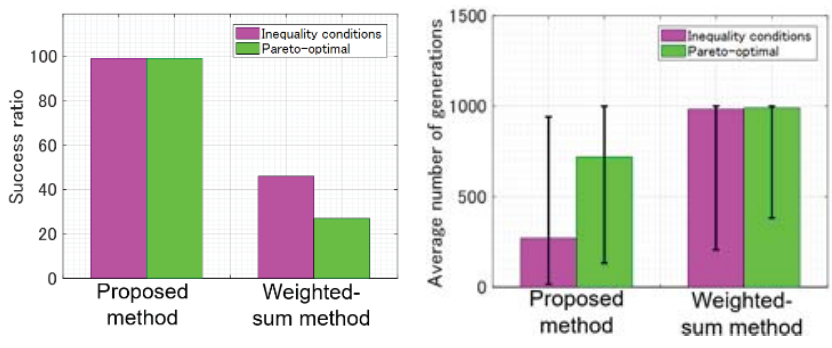

(e) Success ratio in DTLZ7.

Fig. 5. Comparison of the search performance.

We also compared the average calculation times of the solution search processes, as shown in Fig. 6.

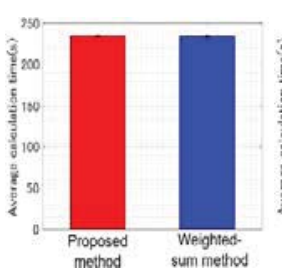

(a) DTLZ1.

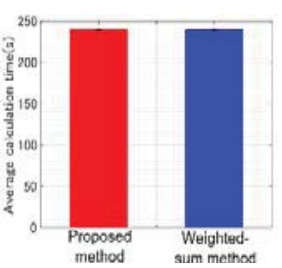

(b) DTLZ3.

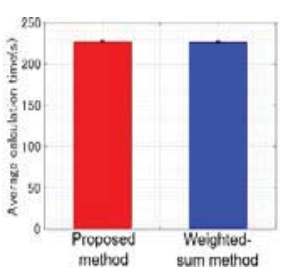

(c) DTLZ7.

Fig. 6. Comparison of the average calculation times.

The red bar represents the average calculation time of the proposed method, and the blue bar represents that of the weighted-sum method. The calculation times of both methods are almost the same for all of the cases. In other words, it can be said that the calculation load is almost the same.

\subsection{Advantages of the proposed method}

The advantages of the proposed method are summarized as follows:

1. There is no need to adjust any parameters such as weighting factors ${ }^{22-24)}$ for solving a multi-objective optimization problem. This shows that applying the proposed method is relatively easy.

2. There is no need to choose a preferred solution from generated solutions because it is obvious that the solution point that is the closest to the ideal point within the aspiration region is the preferred solution for the DM.

3. The proposed method can generate a Pareto-optimal solution that meets constraint conditions efficiently and with high probability compared to the weightedsum method regardless of the shape of the Pareto-frontier or the position of the aspiration point.

4. The calculation load of the proposed method is almost the same as that of the weighted-sum method.

5. The proposed method can be expected to mitigate the effects of the selection pressure problem that occurs in dominance-based methods because the proposed method normalizes all objective function values using corresponding optimal values.

\section{Application to an Aerial Photo-Shoot Mission}

In this section, we attempted to apply the proposed method to a multi-objective path planning problem. It is assumed that the UAV is used in aerial photo-shoot mission for information gathering in the event of a natural disaster. 


\subsection{Flight environment}

The area decomposition method ${ }^{33)}$ was adopted to reduce the computational load. This method divides the terrain and the flight space into a set of three-dimensional 3D cubic cells having the same size. We regarded the $3 \mathrm{D}$ cells that contain the terrain within the cells as "terrain cells", the 3D cells that contain evacuation facilities (EFs) for the victims as "EF cells", and the other cells as "flyable cells", as shown in Fig. 7.

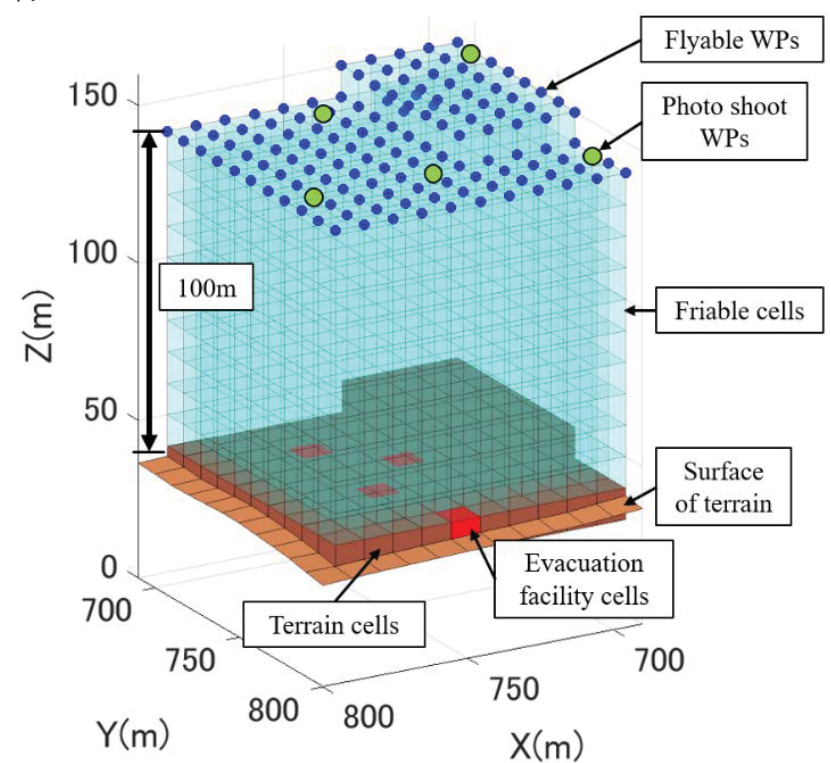

Fig. 7. Differentiation of cells by region decomposition.

The shape of each $3 \mathrm{D}$ cell was assumed a cubic with a size of $10 \mathrm{~m}$. Figure 7 also illustrates the location of "flyable waypoints (WPs)" and "photo-shoot WPs." The flyable WPs that the UAV can pass through are set at the apex of the flyable cells at an altitude of $100 \mathrm{~m}$ from the ground. The photoshoot WPs, which are the location for taking aerial photographs, are chosen from the flyable WPs so as to cover the entire terrain while minimizing the overlapping area of the photo-shoot using a simple genetic algorithm. ${ }^{33)}$ Figure 8 shows a diagram where the aerial photo-shoot WPs are located on the 3D terrain map.

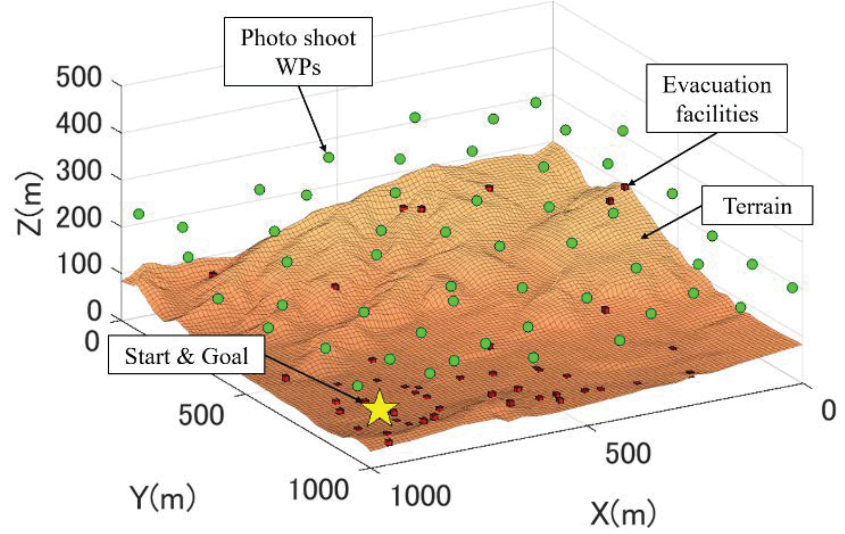

Fig. 8. Placement diagram of aerial photo-shoot WPs.

The terrain is part of a 1-km-square topography in the Tohoku region of Japan. This figure is drawn using some altitude data of the terrain and position data of the EFs provided by the Geospatial Information Authority of Japan. ${ }^{34)}$ The EFs are shown in red and photo-shoot WPs are indicated by green dots. It is assumed that the DM can specify the start and goal point of the mission in advance, and these are shown by the yellow asterisk.

\subsection{Problem statement and calculation conditions}

We assumed that the generated flight path must pass through the preset photo-shoot WPs without overlapping. The flight path must not go through the terrain cells and the EF cells. If there are obstacles present between two photoshoot WPs, a collision-free path must be generated by passing through other flyable WPs. Mitsutake et al. proposed the $\mathrm{A}^{*}$-EC path planning method ${ }^{35)}$ that can generate a three-dimensional suboptimal flight path while considering obstacle avoidance. This method consists of two key steps. First, all of the shortest legs that connect any combination of two WPs while avoiding obstacles are generated using the $\mathrm{A}^{*}$ algorithm. Second, the method optimizes the combination of these legs using EC in order to generate the shortest path. In this second process, we used the proposed method to generate a multi-objective flight path by minimizing not only the flight distance, but also the photo-shoot time of evacuation facility and battery consumption.

Figure 9 is a conceptual diagram that shows part of the generated flight path and the state of the UAV taking an aerial photograph from one of the photo-shoot WPs. As shown in this figure, the angle of view of the UAV's onboard camera is assumed to be 120 degrees.

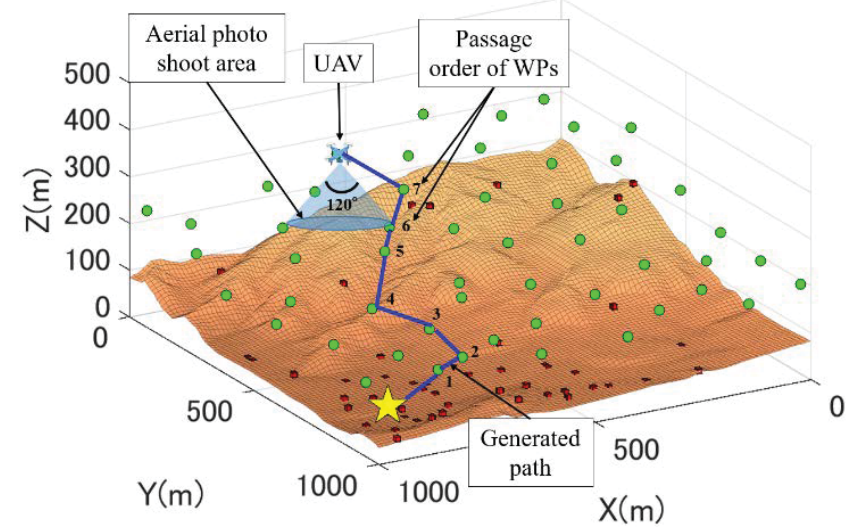

Fig. 9. Schematic of the path planning.

To formulate the path planning problem, we define four new objective functions as follows:

$\mathrm{F}_{1}$ : the total flight distance for completing the photo shoot of the whole terrain.

$$
\mathrm{F}_{1}(\mathbf{x})=\sum_{1=1}^{\mathrm{N}_{W \mathrm{P}}-\mathbf{1}}\left\|\mathbf{W P}_{\mathbf{1 + \mathbf { 1 }}}-\mathbf{W} \mathbf{P}_{\mathbf{l}}\right\|
$$

$\mathrm{F}_{2}$ : the total time for completing the photo shoot of all EFs.

$$
\mathrm{F}_{2}(\mathbf{x})=\sum_{\mathrm{l}=1}^{\mathrm{N}_{\mathrm{F}}-1} \frac{\left\|\mathbf{W} \mathbf{P}_{\mathbf{1 + 1}}-\mathbf{W} \mathbf{P}_{\mathbf{l}}\right\|}{\mathrm{V}_{\text {const }}}
$$

$\mathrm{F}_{3}$ : the total amount of the change in flight altitude during the mission flight.

$$
\mathrm{F}_{3}(\mathbf{x})=\sum_{\mathrm{l}=1}^{\mathrm{N}_{\mathrm{WP}}-1}\left\|\mathrm{Z}_{\mathrm{l}+1}-\mathrm{Z}_{\mathrm{l}}\right\|
$$


$\mathrm{F}_{4}$ : the total amount of the change in flight path angle during the mission flight

$$
\begin{aligned}
\mathrm{F}_{4}(\mathbf{x}) & =\sum_{1=2}^{\mathrm{NWP}_{\mathrm{WP}}-1} \cos \left(\frac{\mathbf{b}_{\mathbf{l}} \cdot \mathbf{c}_{\mathbf{l}}}{\left|\mathbf{b}_{\mathbf{l}}\right| \cdot\left|\mathbf{c}_{\mathbf{l}}\right|}\right) \\
\mathbf{b}_{\mathbf{l}} & =\mathbf{W} \mathbf{P}_{\mathbf{l}}-\mathbf{W} \mathbf{P}_{\mathbf{l}-\mathbf{1}} \\
\mathbf{C}_{\mathbf{l}} & =\mathbf{W} \mathbf{P}_{\mathbf{l}}-\mathbf{W} \mathbf{P}_{\mathbf{l}+\mathbf{1}}
\end{aligned}
$$

$\mathrm{F}_{1}(\mathbf{x})$ is intended to minimize the flight distance for the aerial photo-shoot mission. $F_{2}(\mathbf{x})$ is intended to take photos of the EFs on a priority basis. $F_{3}(\mathbf{x})$ and $F_{4}(\mathbf{x})$ are intended to reduce the consumption of the UAV's battery or fuel. Here, we again compared the performance of the proposed method with the weighted sum method. In the weighted sum method, weighting factors are determined as mentioned in Section 4. The search processes were terminated when the maximum number of generations was reached. This simulation was calculated on a computer with a Core i5-4590 3.30GHz CPU with 8GBytes RAM. C++ was used as a pro-

\begin{tabular}{|c|c|}
\hline Items & Values \\
\hline M & 4 \\
\hline $\mathrm{n}$ & $\begin{array}{l}55 \text { (total number of WPs } \\
\text { including the start and } \\
\text { goal WPs) }\end{array}$ \\
\hline Number of individuals & 100 \\
\hline Mutation probability & 0.01 \\
\hline Crossover probability & 1 \\
\hline Max generation & 5000000 \\
\hline
\end{tabular}
gramming language. The other calculation conditions are shown in Table 3.

\subsection{Simulation results}

First, to generate the flight path mainly minimizing the total flight path distance and the total change in flight path angle, we set $C_{a s p}=[1.3,2.0,2.5,1.3]^{\mathrm{T}}$. Figures 10 and 11 show the respective flight paths generated using these two methods. The influence of the weather and wind are not considered.

At first glance, there are no differences between the two flight paths. To elucidate the difference between these two paths, four graphs that plot each objective function value of the best solution in each generation are shown in Fig. 12. The vertical axis represents each of the dimensionless objective function values, and the horizontal axis represents the

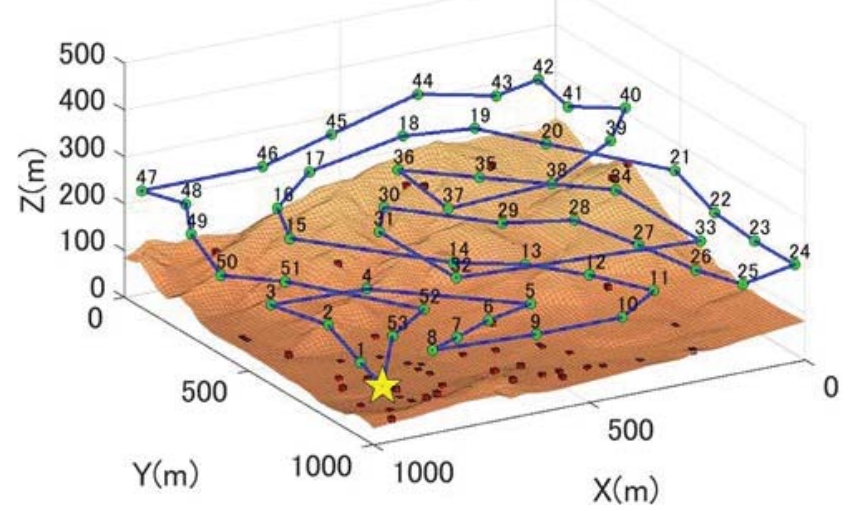

Fig. 10. Flight path determined using the proposed method

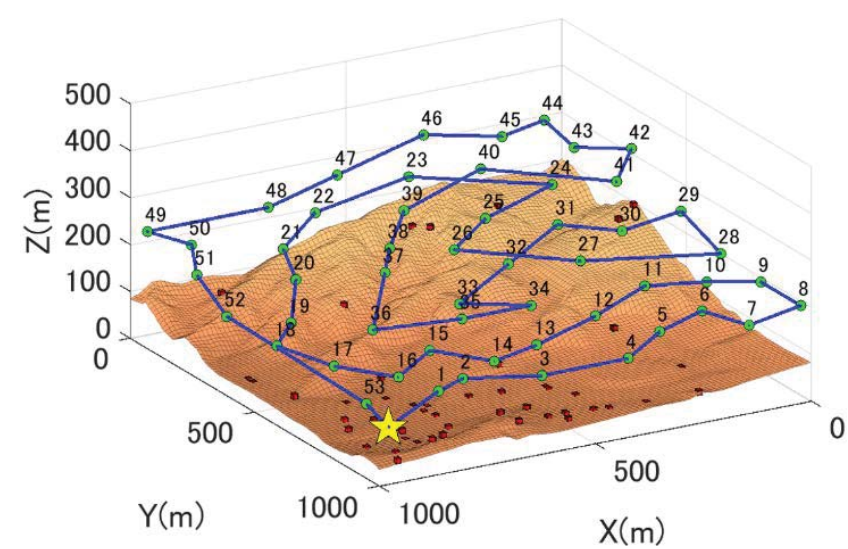

Fig. 11. Flight path determined using the weighted-sum method.

number of generations. The red lines are the best objective function values obtained using the proposed method and the blue lines are that of the weighted-sum method. The black dashed lines represent the constraint conditions. If these values are lower than the lines, it means that the generated solution meets the constraint conditions. Examination of this figure shows that the proposed method generates a solution that satisfies the constraint conditions after 200,000 generations. This is shown at the position of A surrounded by the green circle. The transition of the evaluation value after the generations means updating the position of the aspiration point. It can also be confirmed that the constraint conditions were continually satisfied until the maximum number of generations has elapsed. On the other hand, the calculation of the weighted-sum method ended despite the constraint condition of $\mathrm{F}_{4}^{\prime}(\mathbf{x})$ not being satisfied, as shown at the position of B surrounded by the magenta circle.
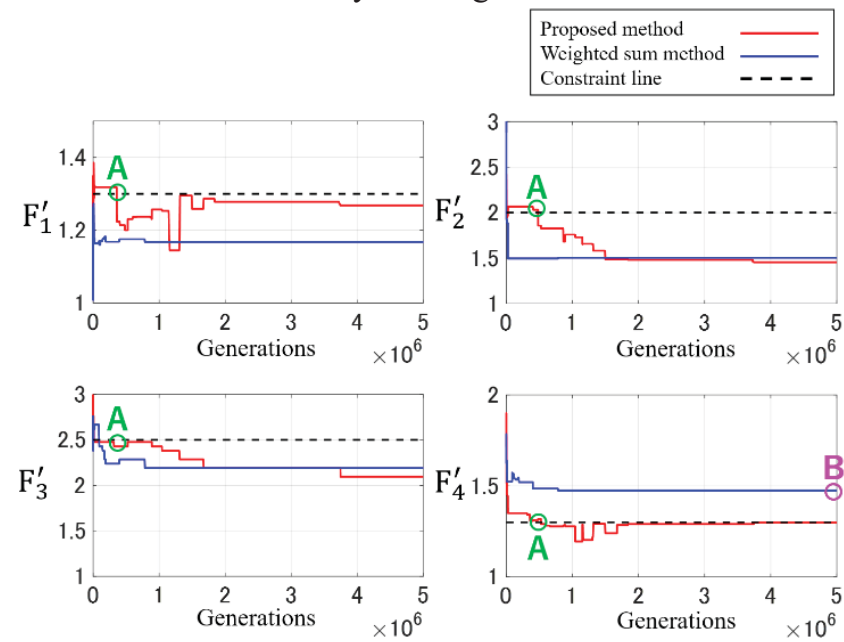

Fig. 12. Comparison of the objective function values.

We attempted to further confirm the usefulness of the proposed method by repeating the same test 100 times. The tests were carried out while varying the position of the aspiration point within the range from 1.3 to 2.3 for each calculation. When neither method can generate a solution that satisfies the constraint conditions, we invalidated the calculation result and performed the tests again after relocating the position of the aspiration point.

Figure 13 (a) shows the success ratio of generating a solution that meets the constraint conditions. The red bars represent the results for the proposed method, and the blue bars 


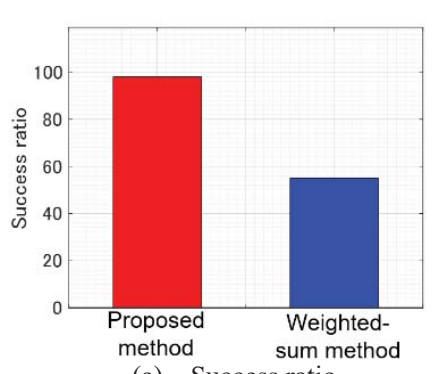

(a) Success ratio.

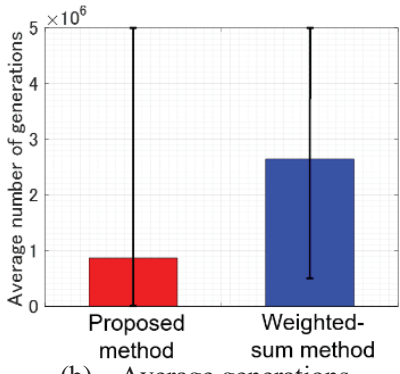

(b) Average generations.

Fig. 13. Comparison of the search performance.

represent those of the weighted sum method. As can be seen from this figure, the success ratio of the proposed method was closer to $100 \%$, whereas that of the weighted sum method was 55\%. Therefore, the proposed method is superior to the weighted sum method in terms of the success ratio.

Figure 13 (b) shows the average number of generations when a solution that meets the constraint conditions was generated. The black vertical lines represent the maximum and minimum number of generations. This figure shows that the weighted-sum method required approximately three times the number of average generations to generate the solution compared to the proposed method.

\section{Conclusion}

In this research, we proposed an aspiration-point-based evolutionary multi-objective optimization method.

The proposed method defines a reference solution that represents the preferred information of the DM named the aspiration point. It also defines a virtual solution, which optimizes all of the objective functions simultaneously, named the ideal point. The generated individuals are evolved toward the aspiration region, which is determined according to the position of the aspiration point. If there is a solution closer to the ideal point than the aspiration point within the aspiration region, the aspiration point is moved to the position of the solution. We can obtain the preferred solution for the DM by repeating this process.

We evaluated the usefulness of the proposed method using the DTLZ benchmark test problems. As a result, it was shown that the proposed method can find the Pareto-optimal solution while meeting the constraint conditions with high probability with the same degree of computational load as the weighted-sum method regardless of the Pareto-frontier shape or the position of the aspiration point.

Finally, we confirmed the practicality of the proposed method by applying it to a multi-objective path planning problem that assumes an aerial photo-shoot mission for gathering information. In this problem, we defined four objective functions such as flight distance, the priority order of aerial photo-shoot and the total amount of the change in flight altitude or path angle. It was found that the proposed method successfully generated a flight path that meets the constraint conditions with high probability and requiring less calculation load compared to the weighted-sum method. Additionally, there is no need to adjust any parameters related to the multi-objective optimization, such as the weighting factors.

\section{References}

1) Gomez, D., De, A. I., and Lopez, F.: Assessing the Accuracy of Mosaics from Unmanned Aerial Vehicle Imagery for Precision Agriculture Purposes in Wheat, Precision Agriculture, 15 (2014), pp. 44-56.

2) Sa, I., Hrabar, S. and Corke, P.: Outdoor Flight Testing of a Pole Inspection UAV Incorporating High-Speed Vision, Field and Service Robotics, Springer International Publishing, (2015), pp. 107-121.

3) Tomic, T., Schmid, K., Lutz, P., Domel, A., Kassecker, M., Mair, E. and Burschka, D.: Toward a Fully Autonomous UAV: Research Platform for Indoor and Outdoor Urban Search and Rescue, IEEE Robotics \& Automation Magazine, 19, (2012), pp. 46-56.

4) Qi, J., Song, D., Shang, H., Wang, N., Hua, C., Wu, C. and Han, J.: Search and Rescue Rotary-Wing UAV and Its Application to the Lushan Ms 7.0 Earthquake, Journal of Field Robotics, 33(3), (2016), pp. 290-321.

5) Maza, I., Kondak, K., Bernard, M. and Ollero, A.: Multi-UAV Cooperation and Control for Load Transportation and Deployment, Journal of Intelligent and Robotic, 57 (2010), pp. 417-449.

6) Thiels, C. A., Aho, J. M., Zietlow, S. P. and Jenkins, D. H.: Use of Unmanned Aerial Vehicles for Medical Product Transport, Air Medical Journal, 34 (2015), pp. 104-108.

7) Danilov, A. S., Smirnov, U. D. and Pashkevich, M. A.: The System of the Ecological Monitoring of Environment Which is Based on the Usage of UAV, Russian Journal of Ecology, 46 (2015), pp. 14-19.

8) Luo, C., Nightingale, J., Asemota, E. and Grecos, C.: A UAV-Cloud System for Disaster Sensing Applications, IEEE 81st Vehicular Technology Conference (VTC Spring), (2015), pp. 1-5.

9) Hart, P. E., Nilsson, N. J. and Raphael, B.: A Formal Basis for the Heuristic Determination of Minimum Cost Paths. IEEE Transactions on Systems Science and Cybernetics, 4(2), (1968), pp. 100-107.

10) Montiel, O., Sepulveda, R. and Orozco, U.: Optimal Path Planning Generation for Mobile Robots Using Parallel Evolutionary Artificial Potential Field, Journal of Intelligent \& Robotic Systems, 79 (2015), pp. 237-257.

11) Bhattacharya, P. and Gavrilova, M. L.: Roadmap-Based Path Planning-Using the Voronoi Diagram for a Clearance-Based Shortest Path, IEEE Robotics \& Automation Magazine, (2008), pp. 58-66.

12) Altiparmak, F., Gen, M., Lin, L. and Paksoy, T.: A Genetic Algorithm Approach for Multi-Objective Optimization of Supply Chain Networks, Computers \& Industrial Engineering, 51(1), (2006), pp. 196-215.

13) Marler, R. T. and Arora, J. S.: Survey of Multi-Objective Optimization Methods for Engineering, Structural and Multidisciplinary Optimization, 26 (2004), pp. 369-395.

14) Deb, K. and Kaisa, M.: Multi-Objective Optimization, Interactive and Evolutionary Approaches, Springer Science and Business Media, 5252, (2008).

15) Nidamarthi, S. and Deb, K.: Muilti-objective Optimization Using Non-Dominated Sorting in Genetic Algorithms. Evolutionary Computation, 2, (1994), pp. 221-248.

16) Deb, K., Pratap, A., Agarwal, S. and Meyarivan, T. A. M. T.: A Fast and Elitist Multi-Objective Genetic Algorithm, NSGA-II. IEEE Transactions on Evolutionary Computation, 6 (2002), pp. 182-197.

17) Zitzler, E., Laumanns, M. and Thiele, L.: SPEA2: Improving the Strength Pareto Evolutionary Algorithm, 2001,

18) Murata, T. and Ishibuchi, H.: MOGA: Multi-Objective Genetic Algorithms, In Evolutionary Computation, IEEE International Conference, 1 (1995), pp. 289.

19) Tapia, M. G. C. and Coello, C. A. C.: Applications of Multi-Objective Evolutionary Algorithms in Economics and Finance, A Survey. In IEEE Congress on Evolutionary Computation, 7 (2007), pp. 532539.

20) Rajesh, J. K., Gupta, S. K., Rangaiah, G. P. and Ray, A. K.: MultiObjective Optimization of Industrial Hydrogen Plants, Chemical Engineering Science., 56 (2001), pp. 999-1010.

21) Ren, H., Zhou, W., Nakagami, K. I., Gao, W. and Wu, Q.: MultiObjective Optimization for the Operation of Distributed Energy Systems Considering Economic and Environmental Aspects, Applied Energy, 87 (2010), pp. 3642-3651. 
22) Kim, I. Y., and De Weck, O. L.: Adaptive Weighted-Sum Method for Bi-Objective Optimization, Pareto Front Generation, Structural and Multidisciplinary Optimization, 29 (2005), pp. 149-158.

23) Christensen, S. S., Agarwal, R., De-Carvalho, E. and Cioffi, J. M.: Weighted-Sum Rate Maximization Using Weighted MMSE for MIMOBC Beamforming Design, IEEE Transactions on Wireless Communications, 7 (2008), pp. 4792-4799.

24) Das, I., and Dennis, J. E.: A Closer Look at Drawbacks of Minimizing Weighted-Sums of Objectives for Pareto Set Generation in Multicriteria Optimization Problems, Structural Optimization, 14 (1997), pp. 63-69.

25) Deb, K.: Nonlinear Goal Programming Using Multi-Objective Genetic Algorithms, Journal of the Operational Research Society, $\mathbf{5 2}$ (2001), pp. 291-302.

26) Deb, K., and Sundar, J.: Reference Point Based Multi-Objective Optimization Using Evolutionary Algorithms, In Proceedings of the 8th Annual Conference on Genetic and Evolutionary Computation, 2006, pp. 635-642.

27) Mohammadi, A., Omidvar, M. N., and Li, X.: Reference Point Based Multi-Objective Optimization through Decomposition, IEEE Congress on Evolutionary Computation, 2012, pp. 1-8.

28) Deb, K., Thiele, L., Laumanns, M., and Zitzler, E.: Scalable Test Problems for Evolutionary Multi-Objective Optimization, Springer London, 2005, pp. 105-145.
29) Ishibuchi, H., Hitotsuyanagi, Y., Tsukamoto, N., and Nojima, Y.: Many-Objective Test Problems to Visually Examine the Behavior of Multi-Objective Evolution in a Decision Space, In International Conference on Parallel Problem Solving from Nature, Springer Berlin Heidelberg. 2010, pp. 91-100.

30) Varadharajan, T. K., and Rajendran, C.: A Multi-Objective Simulated-Annealing Algorithm for Scheduling in Flowshops to Minimize the Makespan and Total Flowtime of Jobs, European Journal of Operational Research, 167 (2005), pp. 772-795.

31) Spears, W. M.: Adapting Crossover in Evolutionary Algorithms, Evolutionary Programming, 1995, pp. 367-384.

32) Poli, R. and Langdon,W. B.: Schema Theory for Genetic Programing with One-Point Crossover and Point Mutation, Evolutionary Computation, 6 (1998), pp. 231-252.

33) Setoguchi, K., Higashino, S. and Mitsutake, K.: Path Planning for Disaster Monitoring by a Small Rotorcraft UAV, Aerospace Technology Japan., 15 (2016), pp. 23-32. (in Japanese).

34) Geospatial Information Authority of Japan.: Base Map Information Download Service, http://fgd.gsi.go.jp/ download/menu.php.

35) Mitsutake, K., and Higashino, S.: An A*-EC Hybrid Path Planning Method for Waypoints Traveling Problem Considering Terrain, AIAA Guidance, Navigation and Control Conference and Exhibits, 2008, pp. 7133. 